\title{
Effect of Low-Energy Nanosecond Laser Therapy on Reparative Osteogenesis in vivo
}

DOI: $10.17691 / \mathrm{stm} 2019.11 .2 .06$

Received May 30, 2018

E.A. Bazikyan, MD, DSC, Professor, Head of the Department of Oral Surgery1;

A.A. Chunikhin, MD, PhD, Associate Professor, Department of Oral Surgery1;

A.G. Chobanyan, PhD Student, Department of Oral Surgery";

E.V. Akhmazov, PhD Student, Department of Oral Surgery';

G.N. Zhuruly, MD, DSC, Professor, Department of Oral Surgery';

M.Y. Sahakyan, MD, DSe, Associate Professor, Head of the Department of Orthopedic Dentistry and Orthodontia2;

O.V. Zayratyants, MD, DSc, Professor, Head of the Department of Pathological Anatomy ${ }^{1}$

Moscow State University of Medicine and Dentistry named after A.I. Evdokimov, 20/1 Delegatskaya St., Moscow, 127473, Russia;

${ }^{2}$ Privolzhsky Research Medical University, 10/1 Minin and Pozharsky Square, Nizhny Novgorod, 603005, Russia

The aim of the study was to assess the effect of low-intensity pulsed laser radiation on reparative osteogenesis of the jaw bones in the in vivo experimental study.

Materials and Methods. The experimental study was performed on 70 mature male Wistar rats whose lower molar was moved using an orthodontic spring for 21 days. A laser device with unique radiation parameters in a nanosecond pulsed mode of radiation with a wavelength corresponding to the oxygen absorption peak in the tissues was used for treatment. In the control group, laser treatment was not applied. To confirm the effect of laser radiation with the selected parameters on the stimulation of bone tissue remodeling, a morphological study was carried out followed by a morphometric study with a quantitative assessment of osteoclasts in the periodontal ligament on the border with the alveolar bone and in resorption lacunae.

Results. Already on the third day of the experiment, on the pressure side in the experimental group there was noted dilation and pronounced hyperemia of the periodontal ligament vessels, the appearance of a large quantity of osteoclasts on the border with the alveolar bone as compared to the control group. On day 7, the activation of fibroblasts, osteoblasts, and cementoblasts with the formation of new tooth root cement was noted on the pressure side, while a new cement formation in the control group was observed only on day 14 of the experiment. On day 21, in the experimental group, almost completely regenerated bone tissue of the alveoli was noted; in the control group, at this stage of observation, marked diffuse inflammatory infiltration of leukocytes with an admixture of lymphocytes and macrophages surrounding the fragments of the partially resorbed alveolar bone was seen.

Osteoclasts and osteoblasts are involved in the processes of bone tissue remodeling. Acceleration of bone resorption is of greater significance on the medial side in the direction of the tooth movement, whereas the regeneration processes are more important on the distal side. Morphometric examination showed over a 1.5 -fold increase in the number of osteoclasts in the experimental group compared to the control group at all stages of observation.

Conclusion. Application of low-energy pulsed laser radiation with the given parameters accelerates bone tissue remodeling and contributes to neovascularization and filling the periodontal ligament and adjacent alveolar bone tissue with blood.

Key words: low-energy laser radiation; photodynamic effect; nanosecond pulsed laser; bone tissue remodeling; osteoclastogenesis; osteoblastogenesis.

\section{Introduction}

The possibilities of laser radiation influence on biological tissues due to photochemical, photothermal, and photodynamic effects are great. A laser beam is able to penetrate deep into the tissues and, if certain radiation parameters are set, may cause therapeutic and surgical effects without any damage to the adjacent structures. Laser technologies are widely used in different fields of medicine: ophthalmology, otorhinolaryngology, traumatology, gynecology, urology, as well as dentistry and maxillofacial surgery [1-3]. In spite of a large number of laser devices and numerous researches in the field of laser medicine, there are a great deal of the ranges of wavelengths and radiation frequencies the influence of which on a living cell and the organism as a whole is still insufficiently studied. Of special interest is the infrared spectrum in the ultrashort pulsed range of radiation frequencies [4].

The unique properties of laser radiation are determined by its action on oxygen and water in the tissues. Presence of oxygen in all biological media in small concentrations enables it to work as a photoacceptor absorbing laser radiation photons. Low

Corresponding author: Andrey A. Chunikhin, e-mail: docca74@yandex.ru 
oxygen concentrations in the tissues and generation of active forms in a small quantity define the direction of the stimulating effect on the biological photoreaction objects without any sensitizers $[5,6]$.

Since the problems of regulation of bone formation processes are interesting for various fields of clinical medicine: traumatology, rheumatology, oncology, reconstructive surgery, dentistry, and maxillofacial surgery, the possibilities of laser radiation impact on the remodeling of bone tissue and stimulation of reparative osteogenesis continue to be the subject of scientific inquiry $[7,8]$.

To optimize the process of bone tissue remodeling using laser radiation, it is insufficient to investigate its effect on the bone cellular elements and the processes of mineral metabolism in the bone tissue [9]. The development of new laser devices for medical applications with unique parameters of laser radiation facilitates the study of mechanisms and ways of its effect on bone tissue remodeling. The research group of the Department of Oral Surgery from Moscow State University of Medicine and Dentistry named after A.I. Evdokimov has designed a new laser device capable of generating the pulsed radiation with a nanosecond frequency and the wavelength corresponding to the oxygen absorption peak [10, 11].

To apply this device for studying the possibilities of stimulation of bone tissue remodeling is considered to be optimal.

The aim of the study was to assess the effect of lowintensity pulsed laser radiation on reparative osteogenesis of the jaw bones in the experimental study.

\section{Materials and Methods}

A new laser device on semiconductor chips with a wavelength of $1265-1275 \mathrm{~nm}$ corresponding to the oxygen absorption peak and the possibility to generate pulsed radiation in the quasicontinuous mode with 400-2000 ns frequency has been used in the work. The radiation was focused in the optical light guide of $400 \mu \mathrm{m}$ diameter. The radiation power was set within $2.0 \pm 0.06 \mathrm{~W}$.

The experiment was carried out on 70 mature male Wistar rats 10 weeks of age weighing $180-200 \mathrm{~g}$. In animals anesthetized with intramuscular injections of Calypsol ( $40 \mathrm{mg} / \mathrm{kg}$ in the form of a $5 \%$ solution), a model of medial movement of the first mandibular molar using a spring was created in compliance with Kawasaki and Shimizu technique [12]. One end of the spring with a compression power of $40 \mathrm{~N} / \mathrm{cm}^{2}$ was fixed to the first molar with a ligature wire, the other end was attached to the central incisor with a light-cured composite (Figure 1).

The animals were divided into two groups: experimental group $1(n=35)$ and control group $2(n=35)$. In group 1, the area of the mandibular first molar on the mesial and distal sides underwent contactless laser radiation for 7 days from the beginning of the movement

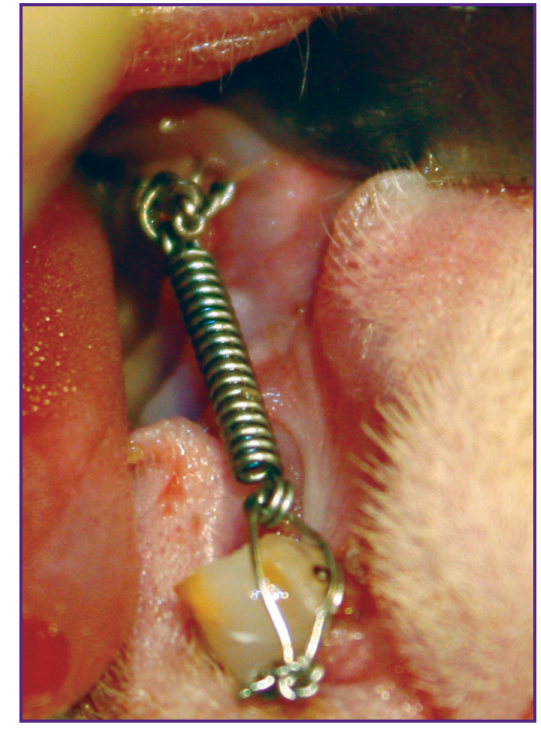

Figure 1. A NiTi spring placed between the molar and central incisor to create the model of tooth movement

keeping a 3-5 $\mathrm{mm}$ focus distance from the radiation site, with $5 \mathrm{~min}$ exposure, and total power of $200 \mathrm{~mJ} / \mathrm{cm}^{2}$. Then after a 7-day interruption, laser radiation exposure was repeated again for 7 days. In animals of group 2 , laser therapy was not conducted.

In order to perform further histomorphological investigations to study the effect of laser radiation on the reparative osteogenesis, 7 animals from each group were withdrawn from the experiment on days $3,5,7,14$, and 21.

The animals were kept and the experiments were conducted in compliance with the Guide for the Care and Use of Laboratory Animals (National Research Council, 2011) and ethical principles of the European Convention for the Protection of Vertebrate Animals used for Experimental and Other Scientific Purposes (Strasbourg, 2006). The study was approved by the Ethics Committee of the Moscow State University of Medicine and Dentistry named after A.I. Evdokimov.

To perform histomorphological investigations, the rat mandible was isolated and separated from the soft tissues, fixed in $10 \%$ neutral formalin for 2 days at $4^{\circ} \mathrm{C}$, and decalcified with Biodec $\mathrm{R}$ reagent (Bio Optica, Italy) according to the manufacturer protocol. After decalcification, a jaw fragment with two first molars was excised and embedded in paraffin following a standard procedure. $3 \mu \mathrm{m}$ thick histological sections were fabricated from the specially oriented paraffin blocks (in order to obtain sections longitudinal to the jaw bone and passing through both molars) on the rotor HM355S microtome (Thermo Scientific, Germany), straightened on the slides and stained with hematoxylin and eosin. The histological specimens were examined and photographed using Axio Lab.A1 microscope (Carl Zeiss Microscopy, Germany). 


\section{BIOTECHNOLOGIES}

Osteoclasts and osteoblasts participate in the process of bone tissue remodeling. Acceleration of bone tissue resorption is of greater importance on the medial side in the direction of the tooth movement, whereas on the distal side, the processes of regeneration are more important. During the morphometric examination, osteoclasts were counted in the microscope visual field on the mesial tooth side. The quantitative assessment was done in the periodontal molar ligament on the border with the alveolar bone in the microscope visual field. Morphological observations of the alveolar bone and periodontal ligament were carried out on the side of pressure and tension. At each stage of observation, 70 morphological specimens were examined on each side viewing 10 fields in each specimen. Thus, 700 fields of the microscopic sections examined under the microscope for each group at each stage of the experiment were used for statistical processing.

The results were processed using computer software, were presented in the form of mean and standard deviation $(\mathrm{M} \pm \sigma)$; nonparametric Mann-Whitney test was used for the assessment. The critical level of significance was accepted equal to 0.05 .

\section{Results and Discussion}

According to the results of the morphological examination, already on day 3 significant differences were noted in the condition of the periodontal ligament of the lower incisors on the border with the alveolar bone in group 1 relative to group 2 on both sides: pressure and tension. On the pressure side, dilation and marked hyperemia of the periodontal ligament vessels (being evidence of reactive inflammation development) and appearance of numerous osteoclasts on the border with the alveolar bone were noted. In the control group, moderate vessel dilation and the appearance of single osteoclasts on the border with the alveolar bone were seen.

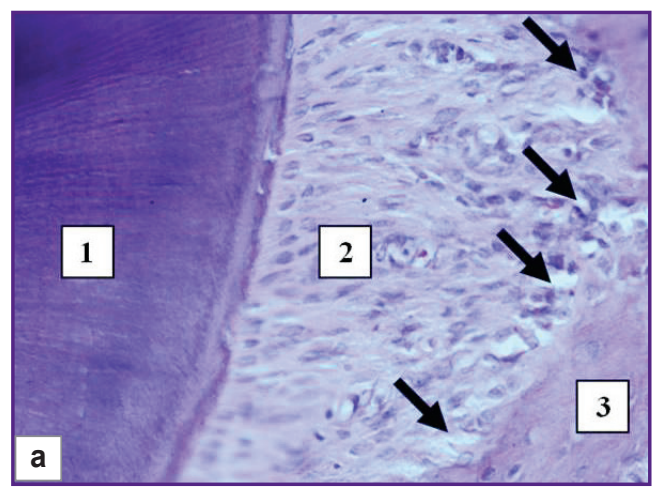

On experimental day 5, proliferation of the small vessels of the periodontal ligament, misalignment of collagen bundles and periodontal ligament edema, increase of osteoclast number in the region of lacunar resorption of the alveolar bone, focal inflammatory leukocyte infiltration with admixture of lymphocytes and macrophages were seen on the pressure side in group 1 (Figure 2 (a)). In the control group, proliferation and widening of the vessel lumens of the periodontal ligament, focal edema of the periodontal ligament, single osteoclasts in the region of lacunar resorption of the alveolar bone, prominent focal and diffuse inflammatory leukocyte infiltration with admixture of lymphocytes and macrophages were observed (Figure 2 (b)).

On day 7 in group 1, activation of fibroblasts, osteoblasts, and cementoblasts with a new root cement formation were detected on the pressure side. In the control group, no significant changes were observed compared to day 5 .

On day 14 , significant increase of osteoclast quantity on the large areas of the alveolar bone lacunar resorption were noted on the pressure side in the experimental group whereas in the control group, activation of fibroblasts, osteoblasts, and cementoblasts, and, for the first time since the beginning of the experiment, formation of a new root cement were noticed at this stage.

On the side of tension at this stage, proliferation of the small vessels and widening of their lumens in the periodontal ligament, activation of fibroblasts, osteoblasts, and cementoblasts with the formation of new root cement, increase in the number of osteoblasts and osteoclasts on the border with the alveolar bone were determined in the experimental group. In the control group, moderate activation of fibroblasts, osteoblasts, and cementoblasts with a focal formation of new root cement as well as a slight increase in the number of osteoblasts and osteoclasts on the border with the alveolar bone were noted.

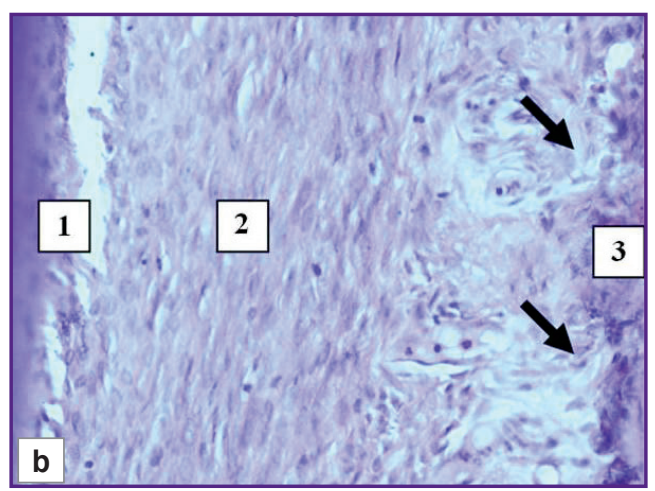

Figure 2. Morphological picture of the examined area after the tooth movement on the side of pressure on experimental day 5 :

(a) in group 1; (b) in group 2; 1 - molar root; 2 - periodontal ligament; 3 - alveolar bone; arrows show the increase in the number of osteoclasts in the areas of lacunar resorption of the alveolar bone; staining with hematoxylin and eosin; $x 400$ 

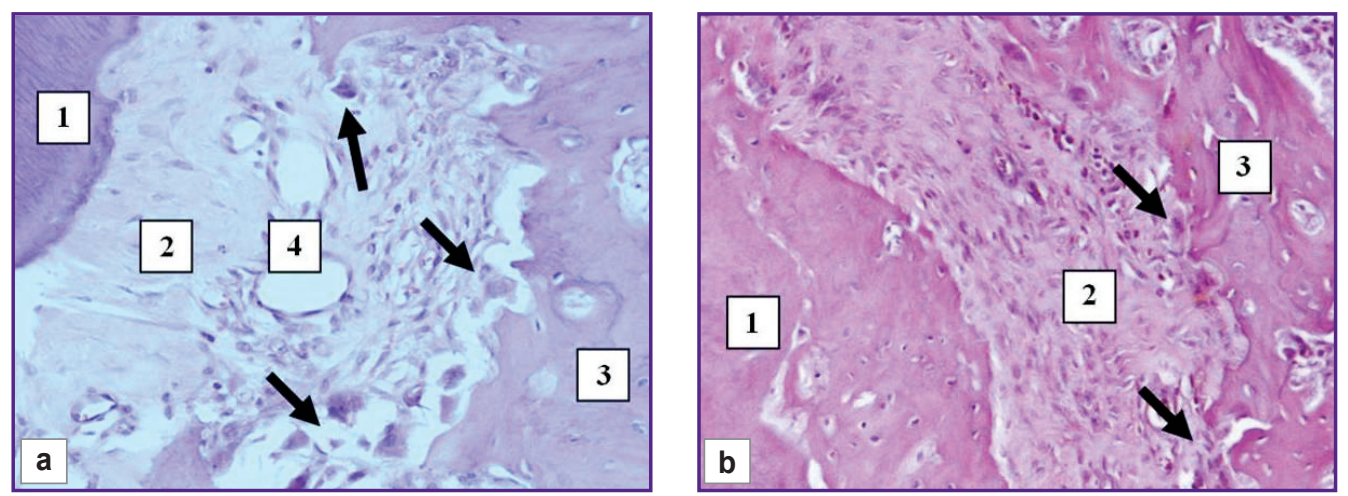

Figure 3. Morphological picture of the examined area after the tooth movement on the side of pressure on experimental day 21 :

(a) in group 1; (b) in group 2; 1 - molar root; 2 - periodontal ligament; 3 - alveolar bone; 4 proliferation of the small vessels and widening of their lumens; arrows show osteoclasts in the areas of lacunar resorption of the alveolar bone; staining with hematoxylin and eosin; x400

Average number of osteoclasts in the periodontal ligament on the pressure side on the border with the alveolar bone at different stages of the experiment $(\mathrm{M} \pm \sigma)$

\begin{tabular}{lccccc}
\hline \multicolumn{1}{r}{ Groups } & \multicolumn{5}{c}{ Experimental day } \\
& 3 & 5 & 7 & 14 & 21 \\
\hline Experimental & $9.70 \pm 3.20$ & $16.20 \pm 2.86^{*}$ & $16.40 \pm 3.21$ & $17.70 \pm 3.68$ & $17.90 \pm 3.32^{*}$ \\
\hline Control & $3.70 \pm 1.34$ & $9.30 \pm 3.59^{*}$ & $11.90 \pm 1.66$ & $12.80 \pm 1.93$ & $13.60 \pm 1.78^{*}$ \\
\hline
\end{tabular}

$\mathrm{N}$ o t e. The difference is statistically significant $(p<0.05)$ between all values of the main and control group and the intergroup values on different experimental days compared to the previous term (shown with asterisks).

On day 21 , on the pressure side, a significant increase of osteoclasts on the large areas of the lacunar resorption of the alveolar bone was registered in the experimental group in comparison with the controls as well as the activation of fibroblasts, osteoblasts, and cementoblasts with the formation and modeling of new root cement, while in group 2, a marked diffuse inflammatory infiltration of leukocytes with the admixture of lymphocytes and macrophages surrounding the fragments of the partially resorbed alveolar bone was observed (Figure 3).

On the side of tension, the formation of new alveolar bone structures, proliferation of the small vessels in the periodontal ligament, activation of fibroblasts, osteoblasts, and cementoblasts with the formation of new root cement, increase in the number of osteoblasts and osteoclasts on the border with the alveolar bone were found at this stage of observation in group 1. In group 2 on the tension side, a moderate activation of fibroblasts, osteoblasts, and cementoblasts with a focal formation of new root cement, misalignment of collagen bundles and periodontal ligament edema, a slight increase in the number of osteoblasts and osteoclasts on the border with the alveolar bone, small foci of the formation of new structures of the alveolar bone were noted at the final stage of the experiment.
The quantitative assessment of osteoclasts in the periodontal ligament on the pressure side (from the medial side in the direction of the tooth movement) on the border with the alveolar bone including the lacunae of its resorption conducted by a morphometric method confirms the qualitative morphological analysis (see the Table).

The data in the table clearly demonstrate that an average number of osteoclasts in the experimental group are greater at all stages of observation and significantly greater at the early stages: 2.5 times greater on day 3 , about 2 times on day 5. It allows us to state that this effect is caused by the influence of laser radiation on osteoclast proliferation and acceleration of bone tissue remodeling.

\section{Conclusion}

The experimental study carried out on animals has proved by means of histomorphological and histomorphometrical investigations that laser radiation with 1265-1275 nm in the nanosecond pulsed mode promotes earlier and marked neovascularization and filling the periodontal ligament and adjacent tissue of the alveolar bone with blood, activation of fibroblasts, osteoblasts, and cementoblasts, earlier and marked 
formation of new structures of the alveolar bone and new tooth root cement. These data are evidence of acceleration of bone tissue regeneration processes under the action of laser radiation with unique parameters making its application perspective for orthodontic treatment and osteoplastic operations in the maxillofacial area. Application of laser therapy will provide the opportunity to reduce the rehabilitation period for patients with bone pathology and, of no less importance, financial expenditure on their treatment.

Study funding. The work was not supported by any financial sources.

Conflicts of interest. The authors have no conflicts of interest to declare.

\section{References}

1. Shakhno E.A. Fizicheskie osnovy primeneniya lazerov $v$ meditsine [The physical basis of the use of lasers in medicine]. Saint Petersburg; 2012.

2. Bazikyan E.A., Chunikhin A.A. Prospects of improvement of minimally invasive laser technologies in photodynamic therapy dental pathologies. Rossiyskiy stomatologicheskiy zhurnal 2016; 20(5): 228-231.

3. Chunikhin A.A., Mitronin A.V. Endodontic treatment of a pulpitis: traditional and modern approaches. Endodontiya today 2009; 4: 3-10.

4. Voronova O.S., Gening T.P., Sysoliatin A.A., Svetukhin V.V. Effect of femtosecond laser radiation on prooxidant and antioxidant status of mice with experimental cervical cancer. Fundamental'nye issledovaniya 2012; 1: 2427.

5. Zakharov S.D., Ivanov A.V. Light-oxygen effect as a physical mechanism for activation of biosystems by quasimonochromatic light (a review). Biophysics 2005; 50(1): S64-S85.
6. Anquez F., El Yazidi-Belkoura I., Randoux S., Suret P., Courtade E. Cancerous cell death from sensitizer free photoactivation of singlet oxygen. Photochem Photobiol 2011; 88(1): 167-174, https://doi.org/10.1111/j.1751-1097. 2011.01028.x.

7. Sirak S.V., Shchetinin E.V., Petrosyan G.G., Gatilo Yu.Yu. Influence of pulse semiconductor laser infrared range on the activity of alkaline phosphatase in experimental uncomplicated fracture of mandible and traumatic osteomyelitis. Kubanskiy nauchnyy meditsinskiy vestnik 2016; 4(159): 106-110.

8. Schwarz F., Sculean A., Berakdar M., Szathmari L., Georg T., Becker J. In vivo and in vitro effects of an Er:YAG laser, a GaAlAs diode laser, and scaling and root planing on periodontally diseased root surfaces: a comparative histologic study. Lasers Surg Med 2003; 32(5): 359-366, https://doi. org/10.1002/lsm.10179.

9. Marques L., Holgado L.A., Francischone L.A., Ximenez J.P.B., Okamoto R., Kinoshita A. New LLLT protocol to speed up the bone healing process - histometric and immunohistochemical analysis in rat calvarial bone defect. Lasers Med Sci 2014; 30(4): 1225-1230, https://doi. org/10.1007/s10103-014-1580-x.

10. Chunikhin A.A., Sahakyan M.Y., Gazhva S.I., Bazikyan E.A. Development of nanosecond laser module built in the robotic multifunctional surgical complex for minimally invasive therapy of maxillofacial area pathology and estimation of its effects on blood plasma. Sovremennye tehnologii $v$ medicine 2016; 8(4): 30-35, https://doi.org/10.17691/ stm2016.8.4.04.

11. Chunikhin A.A., Bazikyan E.A., Pikhtin N.A. A laser unit for photodynamic therapy and robot-assisted microsurgery in dentistry. Technical Physics Letters 2017; 43(6): 507-510, https://doi.org/10.1134/s1063785017060074.

12. Kawasaki K., Shimizu N. Effects of low-energy laser irradiation on bone remodeling during experimental tooth movement in rats. Lasers Surg Med 2000; 26(3): 282-291, https://doi.org/10.1002/(sici)1096-9101(2000)26:3<282::aidIsm6>3.3.co;2-o. 